Table 1, SGRQ total and domains score in all visits; median (IQR) were presented

\begin{tabular}{|l|l|l|l|l|l|}
\hline & Visit 1 & Visit 2 & $P^{*}$ & Visit 3 & $P \#$ \\
\hline $\begin{array}{l}\text { SGRQ } \\
\text { symptoms }\end{array}$ & $69(51-82)$ & $69(55-82)$ & 0.78 & $69(56-82)$ & 0.55 \\
\hline $\begin{array}{l}\text { SGRQ } \\
\text { activity }\end{array}$ & $54(36-67)$ & $50(34-62)$ & 0.045 & $48(32-65)$ & 0.02 \\
\hline $\begin{array}{l}\text { SGRQ } \\
\text { impact }\end{array}$ & $80(60-93)$ & $79(58-93)$ & 0.94 & $79(60-93)$ & 0.77 \\
\hline $\begin{array}{l}\text { SGRQ total } \\
\text { score }\end{array}$ & $64(49-74)$ & $64(45-73)$ & 0.22 & $61(47-72)$ & 0.19 \\
\hline
\end{tabular}

$(*)=$ Compared to visit $1 ; \quad(\#)=$ Compared to visit 1

\section{P22 YIELD OF MICROBIOLOGY SAMPLES TAKEN DURING BRONCHOSCOPY PERFORMED FOR THE DIAGNOSIS OF LUNG CANCER}

doi:10.1136/thoraxjnl-2012-202678.163

SA Rolin, DJ Waine. Plymouth Hospitals NHS Trust, Plymouth, UK

Introduction and Objectives Previous studies have suggested that bronchial lavage does not improve the yield of bronchoscopy if tumour is visible and biopsies and brushings are taken (1). However, no assessment was made of the microbiological yield from lavage samples. The aim of this study was to determine the prevalence and nature of positive bronchial culture in patients presenting with lung cancer.

Methods A retrospective review was conducted of the case notes of all patients between November 2009 and May 2012 who underwent a flexible bronchoscopy for the diagnosis of lung cancer, and were determined by the operator to have either a definite or probable visible malignancy. In all patients BAL had been performed and sent for microbiological investigation in addition to cytology. Type and frequency of culture were analysed, along with the relationship between culture and lung cancer histology, stage, and the performance status of the patient.

Results 95 patients underwent a flexible bronchoscopy at the time of diagnosis within the time period. The majority were male (62\%) with an average age of 70 years (range 31-91). Culture was positive in $37.7 \%$ of samples. $32.5 \%$ of these organisms were gramme negative (mostly Pseudomonas spp. and coliform bacilli), 20.9\% gramme positive (S. pneumoniae, S. aureus, MRSA), 41.9\% Candida spp., and $4.7 \%$ Aspergillus spp. The predominant form of lung cancer was non-small cell (56\%, of which $58.4 \%$ were squamous), the majority of patients having advanced disease ( $92 \%$ stage IIIA-IV) with a good performance status (55.8\% PS 0-1). Patient characteristics, tumour histology, or stage were not significantly different in patients with, or without, positive culture.

Conclusions This study has shown that more than a third of patients investigated for lung cancer had evidence of bronchial colonisation with potentially pathogenic bacteria at the time of diagnosis. This suggests that lavage ought to remain a routine aspect of bronchoscopy for cancer, as identification of bacteria at this early stage might be used to guide the choice of effective antibiotics for the treatment of subsequent pulmonary infections.

1. Waine DJ et al. Am J Resp Crit Care Med 2004; 169(7):A332.

\section{P23 \\ A MULTIDISCIPLINARY INTERVENTION TO REDUCE ANTIBIOTIC DURATION IN LOWER RESPIRATORY TRACT INFECTIONS}

doi:10.1136/thoraxjnl-2012-202678.164

${ }^{1} \mathrm{CD}$ Murray, ${ }^{1} \mathrm{~A}$ Shaw, ${ }^{1} \mathrm{TC}$ Fardon, ${ }^{1} \mathrm{R}$ Smith, ${ }^{1} \mathrm{~S}$ Schembri, ${ }^{2} \mathrm{JD}$ Chalmers. ${ }^{1}$ Ninewells Hospital, Dundee, UK; '2University of Dundee, Dundee, UK

Introduction Prolonged courses of antibiotics in patients with lower respiratory tract infections (LRTI) are common and may contribute to antibiotic related side effects and antibiotic resistance. Prescribing shorter antibiotic courses may be equally effective and associated with fewer side effects. We developed and implemented a multidisciplinary intervention to reduce antibiotic duration in hospitalised patients with LRTI.

Methods This was a prospective before and after evaluation study conducted at Ninewells Hospital, Dundee from November 2011May 2012 (pre-intervention) with post-intervention data collection during June and July. The intervention is scheduled to run until November 2012 but here we present the preliminary results.

The multidisciplinary intervention consisted of automatic stop dates for antibiotics, protocolised antibiotic duration based on national guidelines and ward pharmacist feedback and reminders to stop antibiotics. Data recorded, in addition to length of antibiotic treatment, included underlying diagnosis and suspected antibiotic related side effects.

Results Pre-intervention, there were 281 patients (94 pneumonia, 121 exacerbation of COPD, 24 exacerbation of asthma and 42 LRTI/ bronchitis or other chest infection). The mean duration of antibiotics was 8.3 days (range 1-21) with average by diagnosis of 9.3 days for CAP (range 5-21), 8.5 days for LRTI (3-16), 7.7 days for exacerbation of COPD (1-19) and 6.3 days for asthma (1-10). 31.3\% of patients had a potential adverse effect of antibiotics.

In preliminary data from the post intervention group, there were 97 patients (45 pneumonia, 40 exacerbation of COPD, 12 LRTI/ bronchitis). The mean duration of antibiotic therapy was 6.7 days (range 1-14 days), $\mathrm{p}<0.0001$ compared to pre-intervention. Post intervention duration of treatment for CAP was 7.0 days (1-14), $\mathrm{p}<0.0001$ and for COPD patients was 6.4 days (5-14), $\mathrm{p}=0.0008$ compared to pre-intervention. $16(16.5 \%)$ patients post intervention had antibiotic related adverse effects, $p=0.0005$.

There were 25 (8.9\%) deaths pre-intervention and 7 (7.2\%) deaths post-intervention, $\mathrm{p}=0.6$ suggesting the reduction in antibiotic duration did not result in poorer clinical outcomes. 